\title{
EFEITO DE SOLVENTES SOBRE DÍMEROS DE ASFALTENOS
}

\section{LEANDRO PERRUCI ${ }^{1}$, J.C.ALEXANDRE de OLIVEIRA ${ }^{1}$ e SEBASTIÃO M.P. LUCENA ${ }^{1}$}

${ }^{1}$ Universidade Federal do Ceará, Dept. Eng. Química, Grupo de Pesquisa em Separações por Adsorção - GPSA, Campus do Pici, Bl. 709, 60455-760 Fortaleza, CE, Brazil

E-mail para contato: leperruci@hotmail.com

\begin{abstract}
RESUMO - Um problema comum na indústria de petróleo é a agregação dos compostos mais pesados, conhecidos como asfaltenos. Esse problema resulta num aumento significativo de gastos para a separação, e embora seja comum, ainda há muitas incertezas no comportamento e estrutura dos asfaltenos. Com o objetivo de se obter uma metodologia para avaliar o desempenho de potenciais moléculas dispersantes de asfalteno, foi utilizada dinâmica molecular (Material Studio), em sistemas compostos de modelo ilha de asfalteno (Carauta et al. 2005), e os solventes Tolueno, Heptano, p-(n-dodecil)fenol (DP) sem etoxilação e com duas e quatro etoxilações. O cálculo da interação entre as moléculas permitiu prever os coeficientes de Hildebrand. Os melhores resultados para agentes dispersantes foram encontrados para as moléculas etoxiladas.
\end{abstract}

\section{INTRODUÇÃO}

Os asfaltenos desempenham um papel central na indústria do petróleo. Asfaltenos afetam fortemente a viscosidade do petróleo, que impacta em todas as áreas de exploração. As propriedades dos asfaltenos os tornam particularmente úteis na pavimentação e revestimento de materiais. Os asfaltenos também são de grande importância no que se refere a questões ambientais. A importância do asfalteno é inquestionável. No entanto, há uma complexidade ao abordar o asfalteno. A sua identidade química tem sido tema de diversos trabalhos (Mullins 2010).

Asfaltenos podem ser definidos por seu comportamento na presença de solventes, solúveis em solventes aromáticos, permanecendo insolúvel em compostos alifáticos (Carauta et al. 2005). A fracção de asfaltenos é composta por muitas moléculas de diferentes pesos moleculares e polaridades que têm as mesmas propriedades de solubilidade no solvente. A composição da fracção de asfaltenos não é totalmente conhecida, mas é considerado como um sistema associado de folhas poliaromáticas com diferentes substituições de grupos funcionais (Rogel 1994). Ainda existem algumas dúvidas sobre as interações intermoleculares dos asfaltenos, mas sabe-se que eles se agregam como dímeros.

Existem 3 métodos representativos para investigar o comportamento de desagregação de asfalteno em diferentes solventes, que são: $O$ parâmetro de solubilidade, a distância intermolecular e energia de interação molecular. Neste estudo, o método da distância 


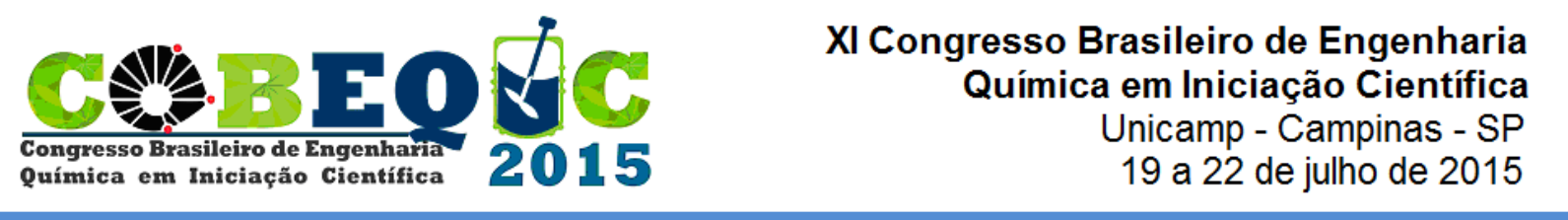

intermolecular foi usado, através da medida da distância entres as moléculas de asfalteno que formam o dímero podemos ver a eficiência de diferentes solventes na desagregação dos asfaltenos (Lu et al. 2008).

O modelo utilizado para representar as moléculas de asfalteno foi desenvolvido no trabalho de Carauta et al. 2005, e para validar o processo da simulação molecular, os dados do comportamento do dímero com tolueno e heptano foram reproduzidos. Feito isso, foi possível desenvolver as simulações com solventes alternativos.

\section{SIMULAÇÃO COMPUTACIONAL}

A simulação molecular foi feita utilizando os módulos Forcite e Amorphous Cell do programa Material Studio. Foi feita a otimização do monômero, utilizando um modelo 3D da molécula de asfalteno proposta no trabalho do Carauta et al. 2005, com 5000 iterações, e usando o método do gradiente conjugado. Após isso, foi feita uma dinâmica molecular de $300 \mathrm{ps}$ a uma temperatura de $300 \mathrm{~K}$. A estrutura de menor energia foi escolhida.

A célula de simulação com o dímero imerso em solvente foi otimizada e após isso, foi realizada uma dinâmica molecular de $100 \mathrm{ps}$, a uma temperatura de $323 \mathrm{~K}$.

Em cada passo da dinâmica, se extraiu um valor de distância entre os centros de massa das moléculas de asfalteno no dímero. Os valores foram acumulados em um banco de dados para posterior construção dos gráficos de distância x tempo de simulação.

\subsection{Modelos Utilizados}

Foram utilizados modelos 3D do asfalteno (Figura 1) e dos seguintes solventes: Heptano, Tolueno, p-(n-dodecil)fenol (DP) (Figura 3), p-(n-dodecil)fenol etoxilatado (2) (DPE2) (Figura 4), p-(n-dodecil)fenol etoxilatado (4) (DPE4) (Figura 5).

Figura 1 - Modelo 3D e molecular do asfalteno utilizado.
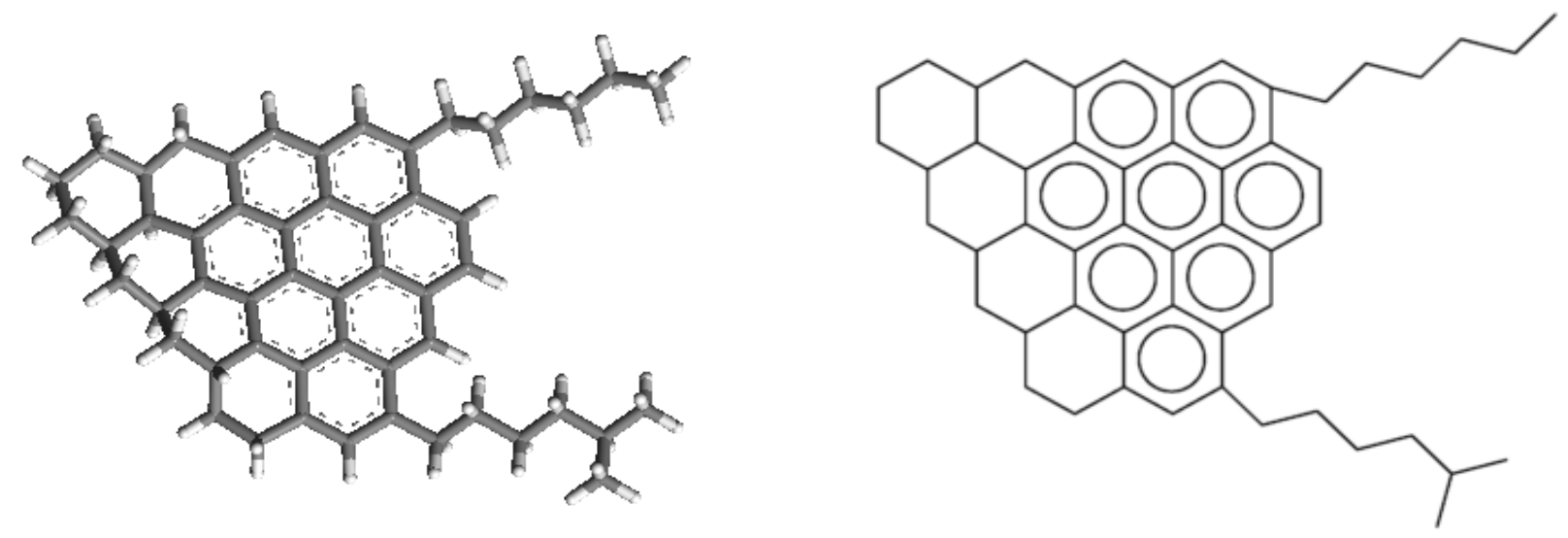


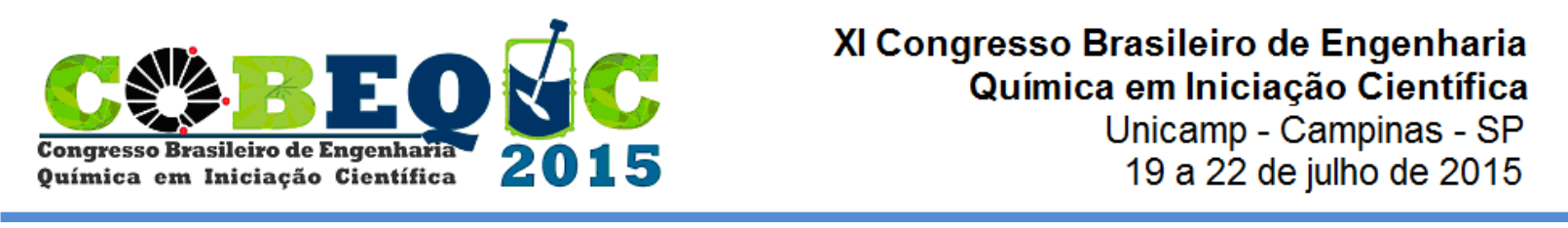

Figura 2 - Dímero de Asfalteno.

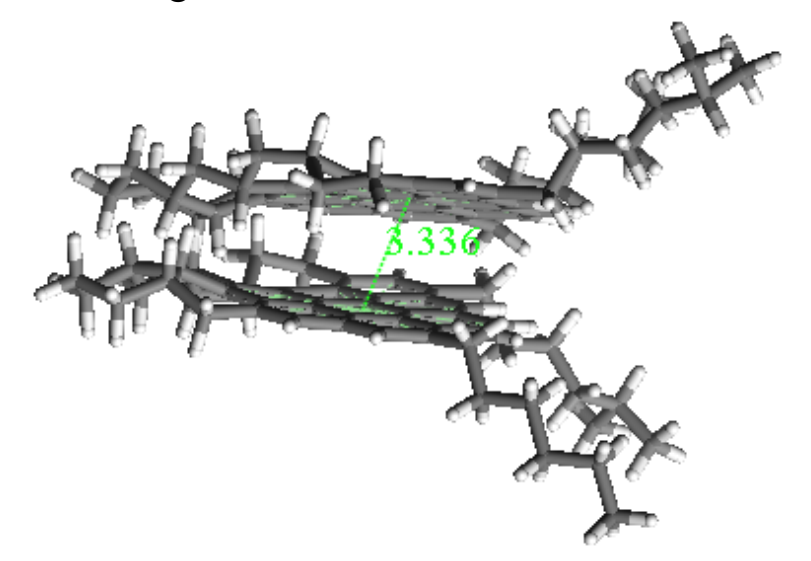

Figura 3 - Modelo 3D DP e fórmula extrutural.
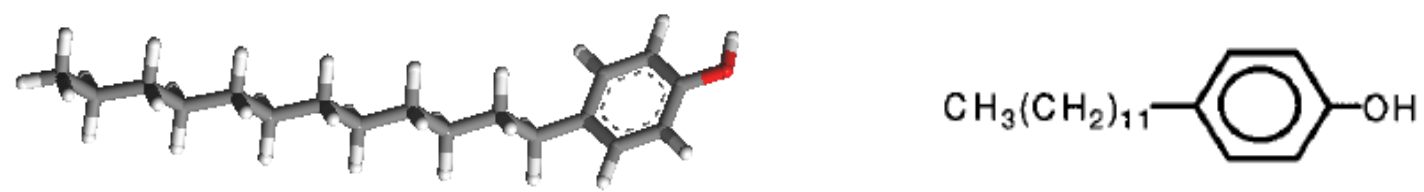

Figura 4 - Modelo 3D DPE2 e fórmula extrutural.
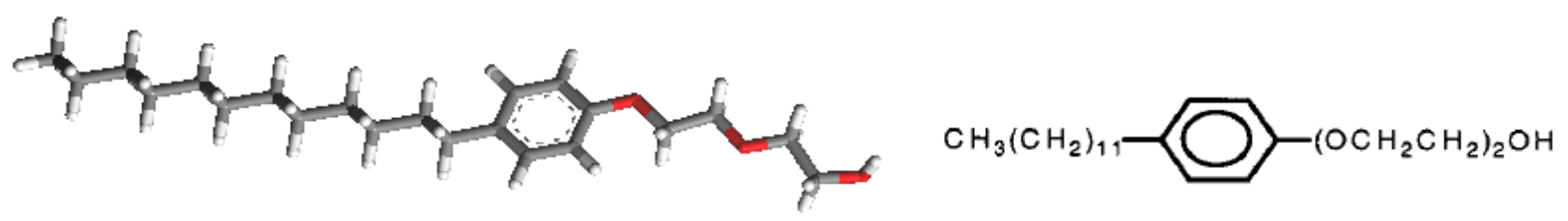

Figura 5 - Modelo 3D DPE4 e fórmula extrutural.

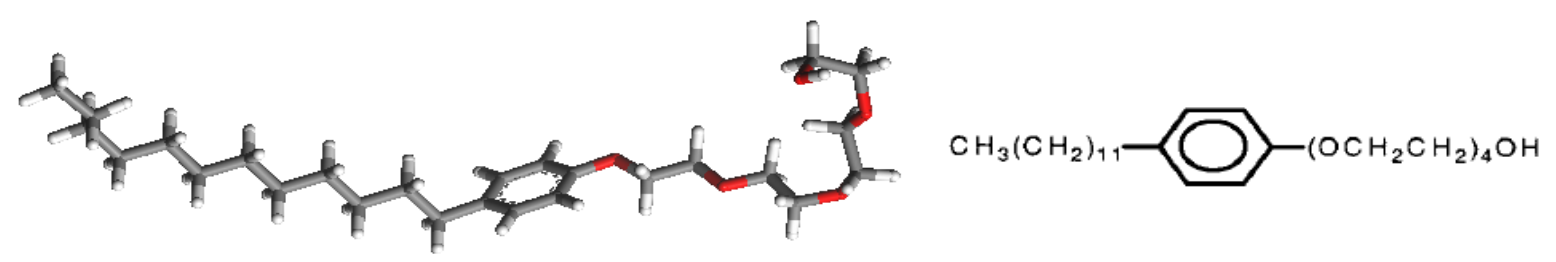

\section{RESULTADOS E DISCUSÃO}

Na Figura 6 comparamos os resultados encontrados por simulação molecular das distâncias entre os monomeros do dímero de asfalteno pelo tempo de simulação com os resultados da literatura (Carauta et al. 2005). Observou-se um concordância dos resultados validando a metodologia utilizada. 


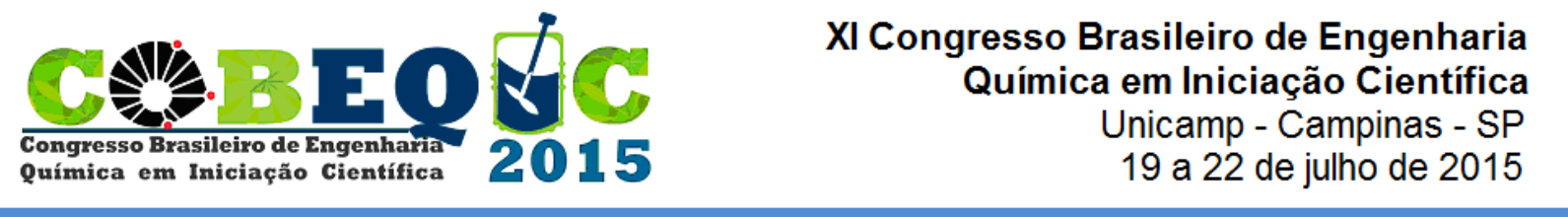

Figura 6 - Comparação entre os resultados obtidos neste trabalho e por Carauta et al., 2005.
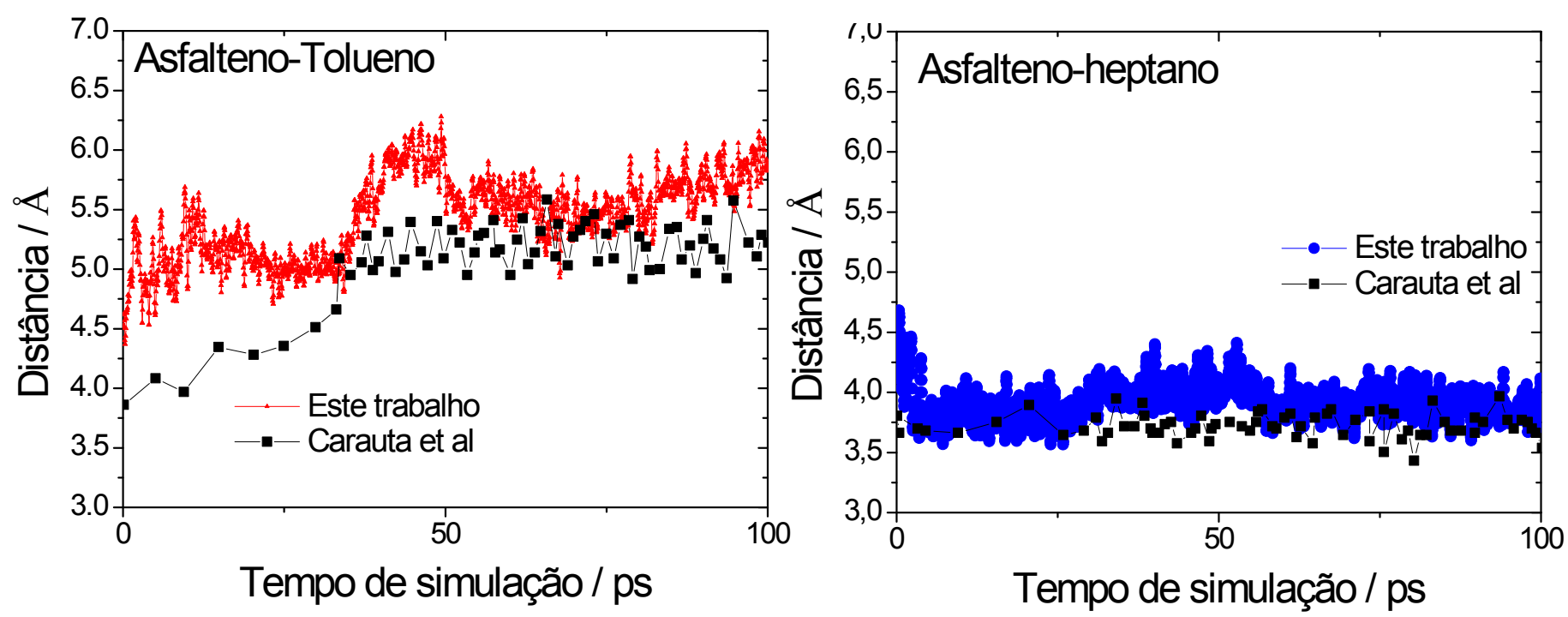

A Figura 7 mostra os resultados das distâncias entre os monômeros de asfalteno no dímero em função do tempo quando o dímero interage com os solventes DP, DPE2 e DPE4. Os dois extremos de solubilidade são observados com esses solventes assim como o tolueno e o n-heptano. Em DP, a distância entre monómeros dificilmente se altera, refletindo a sua

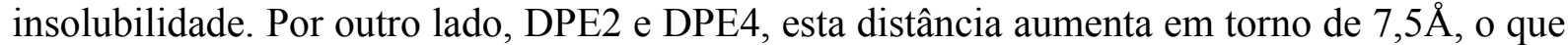
indica que o agregado terá uma tendência para dissolver e dissociar-se, esse resultado pode ser devido aos grupos etoxilados, uma vez que não estão presentes no p-(n-dodecil)fenol (DP). As Figuras $7 \mathrm{~b}$ e $7 \mathrm{c}$ mostram que ao aumentarmos a quantidade de grupos etoxilados não foi o suficiente para ter uma melhor separação no dímero, permanecendo a distância em torno de um mesmo valor de 7,5 $\AA$. Um outro parâmetro para justificar a solubilidade do asfalteno nesse solventes é o coeficiente de Hildebrand que é calculado como a raíz quadrada da densidade de energia coesiva de uma substânica. A Equação 1, representa o cálculo da energia coesiva:

$$
E=\Delta_{v a p} H-R T
$$

Para a densidade de energia coesiva (CED), divide-se a Equação 1, pelo volume de substância, como mostra a Equação 2:

$$
C E D=\frac{E}{V}=\frac{\Delta_{v a p} H-R T}{V}
$$

Finalmente, o coeficiente de Hildebrand $(\delta)$ é dado pela raíz quadrada da densidade de energia coesiva, Equação 3:

$$
\delta=(C E D)^{1 / 2}=\left(\begin{array}{l}
E \\
V
\end{array}\right)^{1 / 2}
$$

Os coeficientes de Hildebrand para o DPE2 e DPE4 são mais próximos ao do asfalteno do que o do DP. O que evidiciamos na tabela 1. 


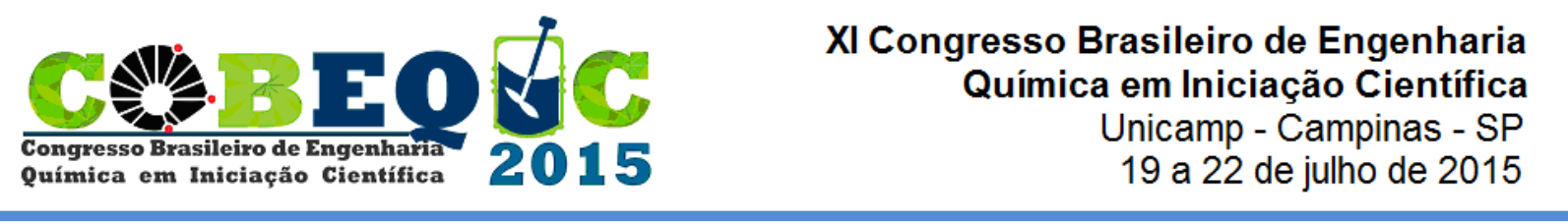

Tabela 1 - Coeficientes de Hildebrand $\left(\mathrm{J} / \mathrm{m}^{3}\right)^{1 / 2}$

\begin{tabular}{ccc}
\hline Composto & $\rho\left(\mathrm{g} / \mathrm{cm}^{3}\right)$ & $\begin{array}{c}\text { Coeficiente de } \\
\text { Hildebrand }\end{array}$ \\
\hline Asfalteno Carauta & 1,2 & 20,16 \\
DP & 0,95 & 18,66 \\
DPE2 & 0,98 & 20,54 \\
DPE4 & 1,27 & 20,08 \\
\hline
\end{tabular}

Figura 7 - Variação da distância entre os as moléculas de asfalteno no dímero, a) DP, b) DPE2 e c) DPE4.
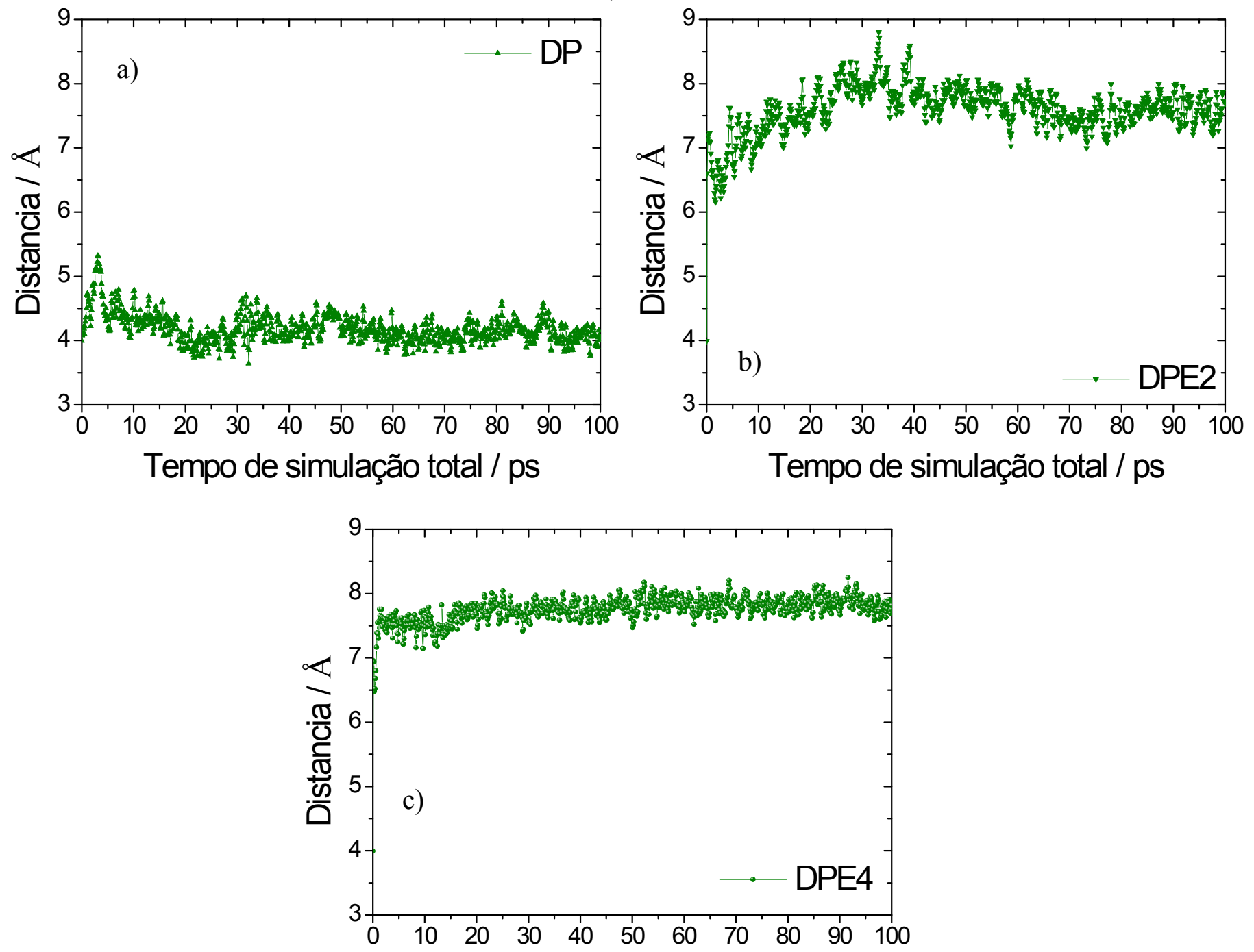

\section{CONCLUSÕES}

Tempo de simulação total / ps 
Dinâmica molecular foi aplicada a um sistema com dímeros de asfalteno para avaliar o grau de dispersão do tolueno, heptano, DP, DPE2 e DPE4. O sistema foi validado para o tolueno e heptano. Evidenciamos que a presença de grupos etoxilados (DPE2 e DPE4) ajuda na solubilização do asfalteno. A técnica de se utilizar dímeros de asfalteno pode efetivamente discriminar o grau de solubilidade de moléculas alvo. O Coeficiente de Hildebrand encontrado foi coerente com relação a variação da distância dos monômeros no dímero. Acreditamos que o método também possa ser aplicado em outras moléculas para avaliar o potencial dispersivo.

\section{REFERÊNCIAS}

Carauta, A.N.M.; Seidl, P.R.; Chrisman, E.C.A.N.; Menechini, D.O.; Silva, D.M., Leal; K.Z., Menezes; S.M.C. De, Souza; W.F. De, Teixeira, M.A.G.: Modeling Solvent Effects on Asphaltene Dimers. Energy \& Fuels. 19, 1245-1251 (2005).

Lu, G., Li, Y.; Song, H., Yu, Y.; Wang, C.: Micromechanism of petroleum asphaltene aggregation. Pet. Explor. Dev. 35, 67-72 (2008).

Lucena, S.M.P.; Frutuoso, L.F. a.; Silvino, P.F.G.; Azevedo, D.C.S.; Toso, J.P.; Zgrablich, G.; Cavalcante Jr., C.L.: Molecular simulation of collection of methane isotherms in carbon material using all-atom and united atom models. Colloids Surfaces A Physicochem. Eng. Asp. 357, 53-60 (2010).

Mullins, O.C.: The Modified Yen Model. Energy \& Fuels. 24, 2179-2207 (2010).

Rogel, E.: Studies on asphaltene aggregation via computational chemistry. Colloids surfaces A Physicochem. Eng. Asp. 104, 85-93 (1994). 